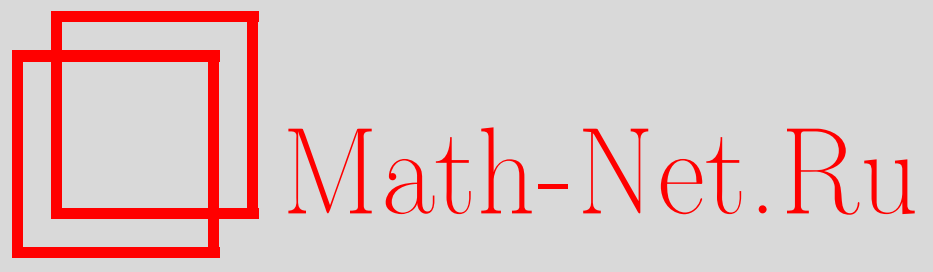

Обперос

В. А. Колмыков, В. В. Меньших, В. Ф. Субботин, М. В. Сумин, Четыре коэффициента характеристического многочлена преобразования Кокстера, Матем. заметки, 2003, том 73, выпуск 5, 788-791

DOI: https://doi.org/10.4213/mzm219

Использование Общероссийского математического портала Math-Net.Ru подразумевает, что вы прочитали и согласны с пользовательским соглашением http://www.mathnet.ru/rus/agreement

Параметры загрузки:

IP : 35.174 .16 .151

26 апреля 2023 г., 13:23:21 


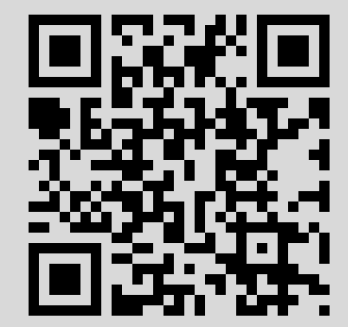




\section{ЧЕТЫРЕ КОЭФФИЦИЕНТА ХАРАКТЕРИСТИЧЕСКОГО МНОГОЧЛЕНА ПРЕОБРАЗОВАНИЯ КОКСТЕРА}

\section{В.А. Колмыков, В. В. Меньших, В.Ф. Субботин, М. В. Сумин}

Мы вычисляем четыре коэффициента характеристического многочлена преобразования Кокстера, связанного с произвольным лесом. Читателю нет необходимости быть знакомым с понятием преобразования Кокстера ${ }^{4}$, достаточно лишь знать, что с каждьм лесом $F$ связан некоторый многочлен, обозначаемый $F(\lambda)$, удовлетворяющий следующим трем свойствам.

1) Многочлен леса равен произведению многочленов его компонент (являющихся деревьями).

2) Для пустого дерева $\operatorname{Emp}$ имеем $\operatorname{Emp}(\lambda)=1$, для дерева $A_{1}$, состоящего из одной вершины, имеем $A_{1}(\lambda)=\lambda+1$.

3) Для дерева $G$ имеет место (см. [2]) формула разрезания

$$
G(\lambda)=G_{2}(\lambda) G_{-2}(\lambda)-\lambda G_{1}(\lambda) G_{-1}(\lambda),
$$

где подграфы $G_{i}$ определяются так. Зафиксируем пару смежных вершин $\alpha$ и $\beta$. Разрушим связь между этими вершинами. Компонента, содержащая $\alpha$, обозначается $G_{-2}$. Другая компонента обозначается $G_{2}$. Лес, получаюшийся из $G_{-2}$ удалением вершины $\alpha$, обозначается $G_{-1}, \mathrm{a}$ лес, получающийся из $G_{2}$ удалением $\beta$, обозначается $G_{1}$.

Теорема 1. а) Многочлен дерева возвратен ${ }^{5}$.

б) Свободный член многочлена дерева равен 1.

в) Коэффичиент при $\lambda$ в многочлене дерева равен 1.

г) Коэффичиент при $\lambda$ в многочлене леса равен числу компонент этого леса.

Идея доказательства. а-в) Индукция по числу вершин + формула разрезания. г) Индукция по числу компонент $+($ б $)+($ в $)$.

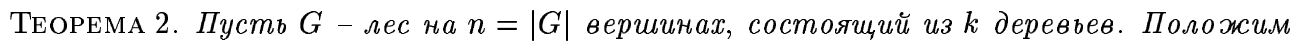

$$
\theta^{(m)}=\sum_{v}(\operatorname{deg} v)^{m}, \quad \tau=\sum_{u G v}(\operatorname{deg} u)(\operatorname{deg} v)
$$

Здесь $\operatorname{deg} v$ - степень вериины $v$, а второе суммирование - по всем неупорядоченным парам смехсных вериин и $и v$.

\footnotetext{
${ }^{4}$ Впрочем, интересующимся мы рекомендуем работу [1], в которой есть все определения и доказано, что все преобразования Кокстера, связанные с лесом, сопряжены и, стало быть, имеют одинаковый характеристический многочлен.

${ }^{5}$ Многочлен $a_{n} x^{n}+\cdots+a_{0}$ называется возвратным, если $a_{i}=a_{n-i}$ для любого $i=0, \ldots, n$.
} 
а) Коэффичиент при $\lambda^{2}$ в многочлене леса равен

$$
2(n-1)-\frac{\theta^{(2)}}{2}+\frac{k^{2}-5 k+4}{2}
$$

б) Коэффичиент при $\lambda^{3}$ в многочлене леса равен

$$
\frac{5}{2} \theta^{(2)}-\frac{10}{3}(n-1)-\frac{1}{3} \theta^{(3)}-\tau+(k-1)\left(2(n-1)-\frac{\theta^{(2)}}{2}\right)+\frac{(k-1)\left(k^{2}-14 k+32\right)}{6} .
$$

СлЕДСтвиЕ. Для графа $G$ через $G^{*}$ обозначим граф, вершины которого находятся во взаимно-однозначном соответствии с ребрами $G$; эти вершинь смежсны тогда и только тогда, когда соответствующ,ие ребра инцидентны (т.е. имеют ровно одну общую вершину) в $G$.

а) Коэффичиент при $\lambda^{2}$ для дерева $G$ равен әйлеровой характеристике (ее определение cм. $в\left[3\right.$, гл. VI, §6]) $\chi G^{*}$ графa $G^{*}$.

б) Коэффичиент при $\lambda^{2}$ для дерева

равен 1 если и только если это дерево чепь,

равен 0 если и только если это дерево трилистник (т.е. имеет ровно одну вершину степени 3, остальные - степени 2 или 1),

меньше нуля в других случаях.

ЗАмечаниЕ. Неформально говоря, преобразование Кокстера - это суперпозиция операторов, являющихся отражениями относительно некоторого набора гиперплоскостей. С этими данньми связано несколько "геометрий". Например, геометрия рассматриваемого пространства и квадратичной формы, инвариантной относительно этих отражений. Другой пример - геометрия алгебр Ли, связанная с первой посредством систем корней. Третий пример - "дискретная геометрия" графа Кокстера, соответствующего рассматриваемому набору отражений. Связь первой и третьей геометрий (являюаяся весьма важной для теории групп, порожденных отражениями, а так же для теории представлений колчанов и модулярных структур) в настоящее время мало изучена. Нас интересует геометрический смысл коэффициентов характеристического многочлена преобразования Кокстера, так как его спектральные свойства (тесно связанные с геометрией графа Кокстера [4], [5]) являются ключевыми в исследовании итераций преобразований и функторов Кокстера [1]. Настоящая заметка показывает, в каком направлении и какой техникой можно искать геометрический смысл этих коэффициентов.

ДоКАЗАТЕЛЬСтво тЕОРЕмЫ 2. а) Коэффициент при $\lambda^{2}$ обозначим $s_{G}$. Из формулы (1) следует, что если $G$ - дерево, то

$$
s_{G}=s_{G_{2}}+s_{G_{-2}}-u-v+1,
$$

где $u=\operatorname{deg} \alpha-1, v=\operatorname{deg} \beta-1$.

В формуле (2) величины $s_{G}, s_{G_{2}}, s_{G_{-2}}$ - инварианты соответствуюших графов, а $u$ и $v-$ нет. Главная идея доказательства состоит в исключении неинвариантных слагаемых.

Имеем

$$
\theta_{G}^{(2)}=\theta_{G_{2}}^{(2)}+\theta_{G_{-2}}^{(2)}+2 u+2 v+2 .
$$

Суммирование (3)+2(2) дает

$$
\theta_{G}^{(2)}+2 s_{G}+4=\left(\theta_{G_{2}}^{(2)}+2 s_{G_{2}}+4\right)+\left(\theta_{G_{-2}}^{(2)}+s_{G_{-2}}+4\right) .
$$

Положим $\psi_{G}=2 s_{G}+\theta_{G}^{(2)}+4$ (например $\psi_{A_{1}}=4$ ). Тогда $\psi_{G}=\psi_{G_{-2}}+\psi_{G_{2}}$, т.е., $\psi$ является аддитивной функцией на деревьях. Из этого следует, что $\psi_{G}=|G| \psi_{A_{1}}=4|G|$. Итак, $2 s_{G}+\theta_{G}^{(2)}+$ $4=4 n$, т.е., $s_{G}=2(n-1)-\theta_{G}^{(2)} / 2$. 
Если $G$ - лес, то коэффициент $s_{G}$ будем искать в виде

$$
s_{G}=2(n-1)-\frac{\theta_{G}^{(2)}}{2}+\nu_{G} .
$$

Пусть лес $G$ состоит из деревьев $G_{1}, \ldots, G_{k}$. Поскольку многочлен леса равен произведению многочленов его деревьев, то

$$
\nu_{G_{1} \cup \ldots \cup G_{m+1}}=\nu_{G_{1}} \cup \ldots \cup G_{m}+m-2 .
$$

Кроме того, $\nu_{G_{1}}=0$. Складывая равенства $\left(4_{m}\right)$ для $m=1, \ldots, k-1$, получаем

$$
\nu_{F_{1} \cup \cdots \cup F_{k}}=\frac{k^{2}-5 k+4}{2}=\frac{(k-4)(k-1)}{2} .
$$

Пункт а) доказан. Отметим, что формулы коэффициента при $\lambda^{2}$ для дерева и четырехкомпонентного леса совпадают (аналогично для двух- и трехкомпонентного леса).

б) Начнем с равенств

$$
\begin{gathered}
\theta_{G}^{(2)}=\theta_{G_{2}}^{(2)}+\theta_{G_{-2}}^{(2)}+2(u+v)+2, \\
\theta_{G}^{(3)}=\theta_{G_{2}}^{(3)}+\theta_{G_{-2}}^{(3)}+3\left(u^{2}+v^{2}\right)+3(u+v)+2, \\
\tau_{G}=\tau_{G_{2}}+\tau_{G_{-2}}+\sum_{\alpha_{i} G_{-2} \alpha} \operatorname{deg} \alpha_{i}+\sum_{\beta_{j} G_{2} \beta} \operatorname{deg} \beta_{j}+(u+1)(v+1),
\end{gathered}
$$

где первое суммирование по всем вершинам $\alpha_{i}$ дерева $G_{-2}$, смежньм с $\alpha$, второе по всем вершинам $\beta_{j}$ дерева $G_{2}$, смежным с $\beta$; как и ранее $u=\operatorname{deg} \alpha-1, v=\operatorname{deg} \beta-1$.

Коэффициенты при $\lambda^{3}$ и $\lambda^{2}$ в многочлене $G(\lambda)$ обозначим соответственно $t_{G}$ и $s_{G}$.

Из формулы (1) следует, что если $G$ - дерево, то

$$
\begin{aligned}
G(\lambda)= & \left(\cdots+t_{G_{2}} \lambda^{3}+s_{G_{2}} \lambda^{2}+\lambda+1\right)\left(\cdots+t_{G_{-2}} \lambda^{3}+s_{G_{-2}} \lambda^{2}+\lambda+1\right) \\
& -\lambda\left(\cdots+s_{G_{1} \cup G_{-1}} \lambda^{2}+(u+v) \lambda+1\right),
\end{aligned}
$$

откуда получаем

$$
t_{G}=t_{G_{2}}+t_{G_{-2}}+\Delta, \quad \text { где } \quad \Delta=s_{G_{2}}+s_{G_{-2}}-s_{G_{1} \cup G_{-1}} .
$$

Далее,

$$
s_{G_{1} \cup G_{-1}}=2\left(\left|G_{1} \cup G_{-1}\right|-1\right)-\frac{1}{2} \theta_{G_{1} \cup G_{-1}}^{(2)}+\frac{1}{2}\left((u+v)^{2}-5(u+v)+4\right)
$$

и

$$
s_{G_{2}}+s_{G_{-2}}=2\left(\left|G_{2}\right|-1\right)-\frac{1}{2} \theta_{G_{2}}^{(2)}+2\left(\left|G_{-2}\right|-1\right)-\frac{1}{2} \theta_{G_{-2}}^{(2)} .
$$

Вычитая (10) - (9), получаем

$$
\begin{aligned}
\Delta=2 & -\frac{1}{2}\left(\theta_{G_{2} \cup G_{-2}}^{(2)}-\theta_{G_{1} \cup G_{-1}}^{(2)}\right)-\frac{1}{2}\left((u+v)^{2}-5(u+v)+4\right) \\
= & 2-\frac{1}{2}\left(2 \sum_{\alpha_{i} G_{-2} \alpha} \operatorname{deg} \alpha_{i}+2 \sum_{\beta_{j} G_{2} \beta} \operatorname{deg} \beta_{j}-\frac{1}{2}\left(u^{2}+v^{2}-u-v\right)\right) \\
& -\frac{1}{2}\left((u+v)^{2}-5(u+v)+4\right) \\
= & -\left(\sum_{\alpha_{i} G_{-2} \alpha} \operatorname{deg} \alpha_{i}+\sum_{\beta_{j} G_{2} \beta} \operatorname{deg} \beta_{j}\right)+3(u+v)-\left(u^{2}+v^{2}\right)-u v .
\end{aligned}
$$


Подставляя это выражение для $\Delta$ в (8), получаем

$$
t_{G}=t_{G_{2}}+t_{G_{-2}}-\left(\sum_{\alpha_{i} G_{-2} \alpha} \operatorname{deg} \alpha_{i}+\sum_{\beta_{j} G_{2} \beta} \operatorname{deg} \beta_{j}\right)+3(u+v)-\left(u^{2}+v^{2}\right)-u v
$$

Складывая $(12)+(7)+\frac{1}{3}(6)-\frac{5}{2}(5)$, получаем

$$
\begin{aligned}
t_{G} & +\tau_{G}+\frac{1}{3} \theta_{G}^{(3)}-\frac{5}{2} \theta_{G}^{(2)} \\
& =t_{G_{2}}+\tau_{G_{2}}+\frac{1}{3} \theta_{G_{2}}^{(3)}-\frac{5}{2} \theta_{G_{2}}^{(2)}+t_{G_{-2}}+\tau_{G_{-2}}+\frac{1}{3} \theta_{G_{-2}}^{(3)}-\frac{5}{2} \theta_{G_{-2}}^{(2)}-\frac{10}{3} .
\end{aligned}
$$

Положим $\phi_{G}=t_{G}+\tau_{G}+\frac{1}{3} \theta_{G}^{(3)}-\frac{5}{2} \theta_{G}^{(2)}-\frac{10}{3}$ (например, $\phi_{A_{1}}=-10 / 3$ ). Тогда $\phi_{G}=\phi_{G_{-2}}+\phi_{G_{2}}$, т.е., $\phi$ является аддитивной функцией на деревьях. Из этого следует, что $\phi_{G}=|G| \psi_{A_{1}}=-\frac{10}{3}|G|$. Этим доказано утверждение для дерева.

Если $G-$ лес, то $t_{G}$ будем искать в виде

$$
T_{G}=\frac{5}{2} \theta_{G}^{(2)}-\frac{10}{3}(|G|-1)-\frac{1}{3} \theta_{G}^{(3)}-\tau_{G}+\gamma_{G}
$$

Если $G_{1}, \ldots, G_{k}$ - полный набор компонент леса $G$, то

$$
\gamma_{G_{1} \cup \cdots \cup G_{m+1}}=\gamma_{G_{1} \cup \cdots \cup G_{m}}+s_{G_{1} \cup \ldots \cup G_{m}}+m s_{G_{m+1}}+\frac{10}{3}
$$

где $\gamma_{G_{1}}=0$;

$$
s_{G_{1} \cup \cdots \cup G_{m}}=s_{G_{1}}+\cdots+s_{G_{m}}+\frac{m^{2}-m}{2} .
$$

Складывая рекурсивные формулы $\left(13_{m}\right)$ для $m=1, \ldots, k-1$, получаем

$$
\begin{aligned}
\gamma_{G} & =(k-1) s_{G}-\frac{(k-1)\left(2 k^{2}-k-20\right)}{6} \\
& =(k-1)\left(2(|F|-1)-\frac{1}{2} \theta_{G}^{(2)}\right)+\frac{(k-1)\left(k^{2}-14 k+32\right)}{6} .
\end{aligned}
$$

ДокАЗАТЕЛЬСтво СЛЕДСтвИЯ. а) Число вершин в $G^{*}$ равно $|G|-1$. Если $d_{1}, \ldots, d_{n}$ - набор степеней вершин дерева $G$, то число ребер в $G^{*}$ равно

$$
\sum \frac{d_{i}\left(d_{i}-1\right)}{2}=\frac{1}{2} \theta_{G}^{(2)}-(|G|-1)
$$

б) Известно, что для связного графа $\chi \leqslant 1$, причем равенство достигается только если этот граф̆ - дерево. Но $G^{*}-$ дерево, только если $G-$ цепь. Далее, $\chi=0$, если и только если в графе ровно один цикл. Но в $G^{*}$ ровно один цикл, если и только если $G$ - трилистник. 


\section{СПИСОК ЦИТИРОВАННОЙ ЛИТЕРАТУРЫ}

1. Бернштейн И. Н., Гельфанд И. М., Пономарев В. А. // УМН. 1973. Т. 28. № 2(170). С. 19-33. 2. Субботин В.Ф., Сумин М. В. Исследование многочленов, связанных с представлениями графов. Воронеж: Воронеж. гос. ун-т, 1982. Деп. в ВИНИТИ 30.12.82, № 6480-82 Деп. 3. Масси У., Столлингс Дж. Алгебраическая топология. Введение. М.: Мир, 1977. 4. Субботин В.Ф., Стекольщик Р. Б. // Функц. анализ и его прилож. 1978. Т. 12. №1. С. 84-85. 5. Ringel C. M. // Math. Ann. 1994. № 300. P. 331-339.

Воронежский государственньй университет (В. А. Колмыков) Воронежский институт МВД России (В.В. Меньших)

E-mail: kolmykov@vak.vsu.ru 